\title{
ИММЕРСИВНЫЕ ТЕХНОЛОГИИ КАК ЧАСТЬ НОВОЙ ОБРАЗОВАТЕЛЬНОЙ РЕАЛЬНОСТИ И ИХ ПРИМЕНЕНИЕ В ОБЩЕОБРАЗОВАТЕЛЬНОЙ ШКОЛЕ
}

\section{IMMERSIVE TECHNOLOGIES AS PART OF NEW EDUCATIONAL REALITY AND THEIR APPLICATION IN SECONDARY SCHOOL}

\section{B. Karev \\ V. Prokoptsev}

Summary: The article presents an analysis of immersive technologies as innovative educational tools, both from the point of view of identifying their essential (common) features, and establishing the species differences of technologies of this kind used in training; immersive technologies are considered in the context of technological support of the educational process when implementing basic educational programs of basic and secondary general education.

Keywords: virtual reality, augmented reality, mixed reality, immersive technologies, basic educational programs of basic and secondary general education.
Карев Борис Анатольевич профессор, член-корреспондент РАО, Российская академия образования (2. Москва) karevdok.27@mail.ru

Прокопцев Владимир Олегович к.т.н., дочент, Хабаровский институт инфокоммуникаций azp_prokoptsev@mail.ru

Аннотация: В статье представлен анализ иммерсивных технологий как инновационных образовательных средств, как с позиции выявления их сущностных (общих) признаков, так и установления видовых отличий технологий такого рода, используемых в обучении; иммерсивные технологии рассмотрены в контексте технологического обеспечения образовательного процесса при реализации основных образовательных программ основного и среднего общего образования.

Ключевые слова: виртуальная реальность, дополненная реальность, смешанная реальность, иммерсивные технологии, основные образовательные программы основного и среднего общего образования.
$\mathrm{P}$ оссия сегодня - это общество, в приоритете которого находится цифровизация различных областей жизни, и это - объективная реальность, определяемая государственной политикой, в том числе и в образовании различного уровня. Последовательная реализация национальных проектов, государственных программ и иных принятых инициатив позволит России в ближайшем будущем перейти на более высокий технологический уклад, предполагающий цифровую экономику. Это невозможно без создания системы образования, модернизированной в формате цифровой среды, которая предполагает использование особых образовательных средств, в том числе и на основе иммерсивных технологий.

Эта тенденция вселяет надежду на возможное создание единого цифрового образовательного пространства в Российской школе всех уровней, однако она требует научного осмысления, мониторинга и научно-педагогического сопровождения, как необходимых условий для рациональной и эффективной ее организации.

Применение иммерсивных технологий в общем образовании является одним из ведущих эволюционных направлений в области совершенствования образовательных средств основного и среднего общего обра- зования, которое предполагает включение учащихся в цифровой учебный процесс за счет создания вспомогательных реальностей (дополненной, виртуальной, смешанной).

Внедрение иммерсивных технологий в общеобразовательную школу согласуется с условиями реализации основной образовательной программы основного и среднего общего образования, одним из которых является «использование в образовательной деятельности современных образовательных технологий» [1, 2]. Согласно федеральным государственным образовательным стандартам основного общего образования и среднего общего образования в школе должна обеспечиваться возможность «...проведения наблюдений и экспериментов, В том числе с использованием учебного лабораторного оборудования цифрового (электронного) и традиционного измерения, виртуальных лабораторий, вещественных и виртуально-наглядных моделей и коллекций основных математических и естественно-научных объектов и явлений», которая предполагает применение в том числе и технологий такого рода.

Под иммерсивными технологиями понимают технологии, предполагающие взаимодействие человека с пространством и информацией в средах разной степе- 
ни виртуальности, созданных посредством электронных устройств. Задача таких технологий - стереть грани между реальным и вымышленными мирами, создать в некотором смысле новую реальность.

Большая часть «реальностей» берет свое начало из компьютерных игр, поскольку именно в них сюжетные линии дополняются пояснительными текстами, схемами, картинками, аудио- и видеоизображениями, к которым может обратиться персонаж (герой игры) с целью получения необходимых знаний для решения игровой задачи.

Другими словами, компьютерные игры предопределили возникновение нового формата в обучении - виртуальной реальности. Под виртуальной реальностью понимается смоделированный искусственный мир, который человек воспринимает через органы чувств. Аналогично компьютерным играм создается эффект трехмерного окружения. Во вновь созданном пространстве пользователь взаимодействует с объектами и предметами, а не с их проекциями.

Рассмотрим примеры технологий, позволяющих реализовать иммерсивное обучение в общеобразовательных организациях.

Виртуальная реальность (Virtual Reality, VR). Эта технология использует цифровое моделирование для воссоздания реальных сценариев. Применяя различные аксессуары (например, очки виртуальной реальности), пользователь может погрузиться в любой созданный виртуальный мир. В качестве иллюзорного мира может выступать как ограниченная территория (например, помещение), так и безграничное пространство. Одной из особенностей виртуального мира является то, что человек обозревает пространство вокруг себя на 360 градусов.

Дополненная реальность (Augmented Reality, AR). Данная технология позволяет дополнить окружающую действительность цифровыми объектами с целью увеличения информативности восприятия реального мира. Виртуальные объекты буквально «накладываются» на реальное пространство, превращая последнее в своего рода цифровой интерфейс. Другим вариантом реализации технологии можно считать применение в дидактических материалах специальных маркеров, считываемых смартфонами через специальные приложения, в результате которого плоские объекты будут превращаться в объемные на экране цифрового устройства.

Обе рассмотренные технологии имеют существенные отличия, но области их применения схожи. B VR отсутствует реальный мир, а пользователь полностью погружается в цифровую среду, которая «реагирует» на действия пользователя. В AR пользователь (человек) смотрит на реальный мир, но с дополнительными элементами - объектами, звуками, маркерами, чертежами и т.д. В отличие от дополненной реальности, для взаимодействия с виртуальной реальностью, кроме смартфона или аналогичного устройства понадобятся специальный шлем или очки.

Смешанная реальность (Mixed Reality, MR) - это технология, позволяющая комбинировать дополненную и виртуальную реальности, которая делает виртуальные взаимодействия более реалистичными. Технология объединяет AR и VR. Создается ощущение, что вы присутствуете в реальном мире с физическими объектами, но вокруг существуют и цифровые объекты, с которыми можно взаимодействовать.

Различия в технологиях AR и MR в том, что в первой объекты представлены рисунками, картинками или голограммами и наложены поверх физических объектов, а во второй цифровые объекты представляют собой неотъемлемую часть реальной обстановки, и при этом имеют физические и пространственные точки соприкосновения с реальными объектами.

Стоит отметить, что с каждым годом приобщиться к виртуальному миру становится все проще, поскольку стоимость устройств начального уровня, минимально необходимого для реализации технологий данного рода уменьшается, и, следовательно, становится доступным, для все большего количества пользователей.

Следует подчеркнуть, что количество вариантов применения иммерсивных технологий в образовании значительно. В настоящей статье рассмотрены лишь некоторые из них в качестве примеров. Так, рассматривая понятия о пространственных геометрических фигурах при изучении геометрии (базовый уровень), возможно применение в качестве образовательного средства технологии дополненной реальности, которая позволяет превращать плоские предметы в объемные посредством цифрового устройства. Применение дополненной реальности актуально в данном случае, так как способствует развитию пространственного мышления у обучающихся.

Применение иммерсивных технологий при изучении физики позволить визуализировать сложные для понимания учащихся явлений (процессов), таких как теплопроводность, конвекция, взаимодействие электрических зарядов, распространение электромагнитных полей и ряд других. Проведение экспериментов (опытов) с указанными явлениями (процессами) в общеобразовательных школах зачастую невозможно в реально- 
сти, поскольку для их реализации необходимо дорогое лабораторное оборудование для регистрации параметров. Использование иммерсивных технологий в столь сложных экспериментах позволит наглядно визуализировать процессы и явления и сопоставить результаты при изменении исходных параметров. Кроме того, каждый из полученных результатов эксперимента (опыта) может быть сохранен и при необходимости использован в дальнейшей работе.

Современный процесс обучения характеризуется большим количеством отвлекающих факторов. И главным из них уже традиционно считают смартфон. Но при правильном подходе и смартфон можно полноценно встроить в процесс обучения, тем самым подменив его функцию «развлечения» на функцию «обучения». Наиболее простым вариантом использования смартфона при внеурочной деятельности является организация виртуальных экскурсий по достопримечательностям: музеи и различные галереи, знаковые исторические и культурные места. Для более полного погружения в «реальность» одним смартфоном уже не обойтись - дополнительными элементами могут стать очки и шлемы VR. Эти дополнительные элементы позволяют весь фокус направить на погружение в виртуальный мир, исключив все сторонние раздражители. Таким образом, обучающихся можно «переместить» непосредственно в тот мир, изучить который им необходимо. А при отсутствии «раздражителей» процесс получения новых знаний становится более сосредоточенным, целенаправленным и, соответственно, увлекательным, что позволяет обучающимся в смоделированной обстановке качественнее усвоить учебный материал.

Кроме того, преимуществом применения иммерсивных технологий в образовании можно считать индивидуальный подход к обучающемуся. Использование технологий «реальностей» позволяет персонализировать процесс обучения, то есть корректировать траекторию развития отдельного обучающегося. Так появляется возможность осваивать тему (учебный предмет) в своем собственном ритме, при необходимости многократно возвращаясь к неусвоенному материалу в любое время. Кроме этого, в виртуальные миры можно встраивать различные голосовые ассистенты и/или движущиеся персонажи с таким вариантом поведения, к которому обучающемуся необходимо стремиться.

На наш взгляд принципиально важно осознание того, что так называемые «предметные результаты освоения основной образовательной программы» характеризу- ются в некотором смысле трехмерностью и включают в себя помимо собственно знаний по определенной предметной области, также опыт отношений и опыт познавательной деятельности, что подразумевает реализацию обучающей, воспитательной и развивающей целей. И только научившись самостоятельно выдвигать конструктивные, созидательные цели и отбирать адекватные им технологии, по сути, став субъектом системы самообразования, обучающийся способен осуществлять качественное развитие социально востребованных сторон своей личности [3].

Внедрение в образовательное пространство иммерсивных технологий предоставляет возможность обучающемуся соотносить достижения своей деятельности с поставленными целями и задачами, видоизменять технологическое обеспечение процесса для получения максимально эффективного результата, что возможно для воплощения, благодаря тому что познание проходит в относительно безопасной нереальной среде. Создание условий, при которых есть возможность «начать сначала» с момента, определенного самим обучающимся, придает последнему уверенности в принятии решении и является мощным стимулом повышения активности в творчестве, развивая тем самым креативное мышление.

Однако, важно чтобы у обучающегося не создавалась иллюзия того, что в реальной жизни всегда будет возможность повторить что-либо и (или) поменять параметры условий для осуществления чего-либо. Именно поэтому применение иммерсивных технологий должно проходить при однозначном понимании индивидом их познавательных возможностей с одной стороны и принципиальных отличий от реальной действительности с другой. Кроме того, внедрение технологий такого рода требует от обучающегося высокой степени ответственности за принятие того или иного решения, так как технологические просчеты в настоящем мире могут иметь весьма отрицательные последствия как для самого индивида, так и для окружающей его действительности.

Востребованность, значимость и масштабность иммерсивных технологий в образовании, опосредованные экономической, политической и социальной целесообразностью, не вызывают сомнений. Однако, следует помнить, что иммерсивные технологии как средство в образовательной деятельности находятся лишь на начальном этапе своего развития. Вот почему именно сейчас необходимо провести анализ их педагогической ценности, чтобы в дальнейшем предотвратить негативные последствия от их некорректного применения. 


\section{ЛИТЕРАТУРА}

1. Приказ Министерства образования и науки РФ от 17 декабря 2010 г. N 1897 «0б утверждении федерального государственного образовательного стандарта основного общего образования» (сизменениями и дополнениями).

2. Приказ Министерства образования и науки РФ от 17 мая 2012 г. N 413 «06 утверждении федерального государственного образовательного стандарта среднего общего образования» (с изменениями и дополнениями).

3. Лернер, И.Я. Дидактические основы методов обучения. М.: Педагогика, 1981. - 186 с.

○ Карев Борис Анатольевич (karevdok.27@mail.ru), Прокопцев Владимир Олегович (azp_prokoptsev@mail.ru).

Журнал «Современная наука: актуальные проблемы теории и практики»

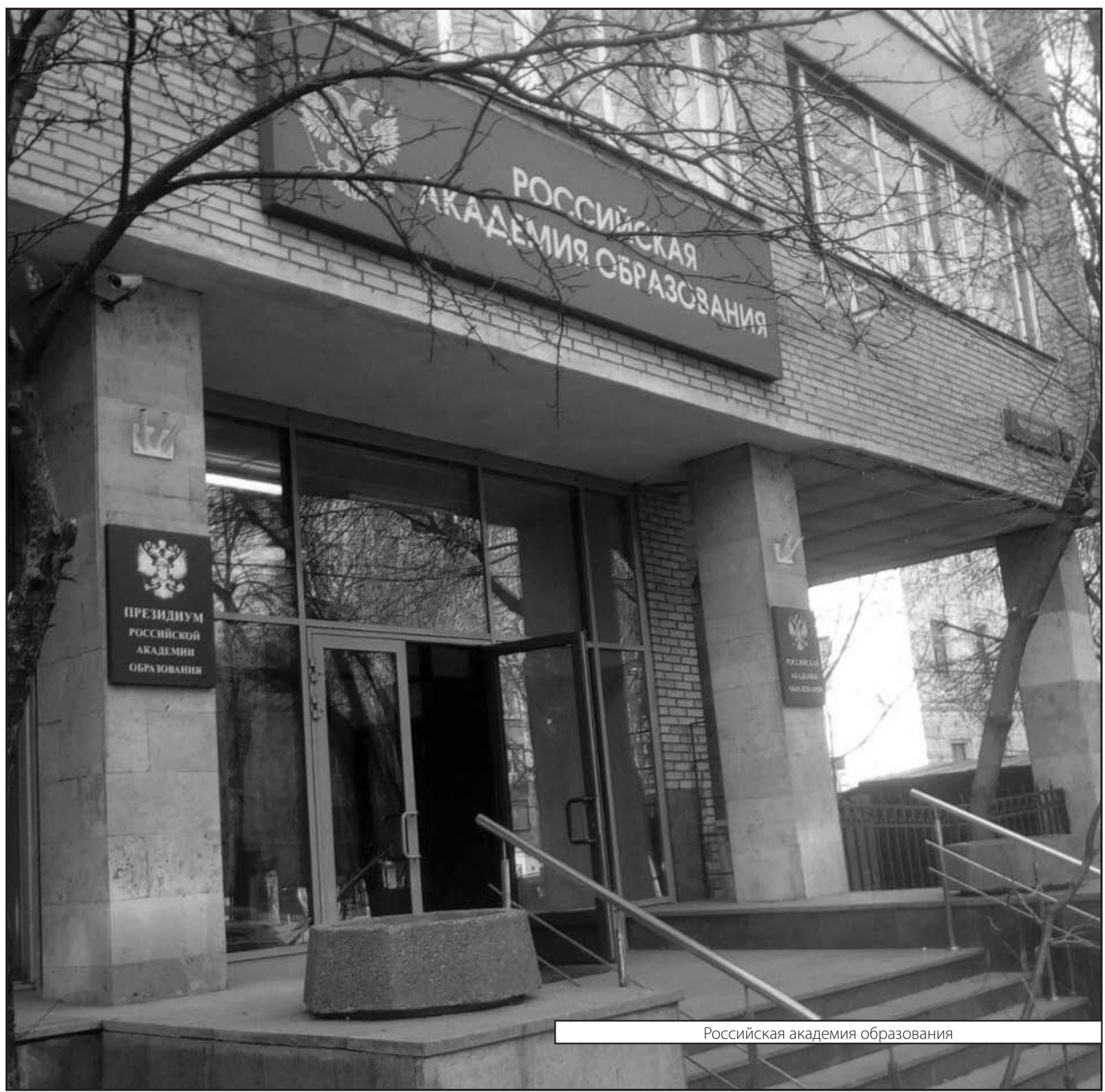

